DPNU-93-01

KUNS-1178

March, 1993

\title{
Proving the Low Energy Theorem of Hidden Local Symmetry
}

\author{
Masayasu HaradA \\ Department of Physics, Nagoya University, \\ Nagoya 464-01, Japan \\ Taichiro Kugo \\ Department of Physics, Kyoto University, \\ Kyoto 606-01, Japan \\ Koichi YAMAWAKI \\ Department of Physics, Nagoya University, \\ Nagoya 464-01, Japan
}

\begin{abstract}
Based on the Ward-Takahashi identity for the BRS symmetry, we prove to all orders of the loop expansion the low energy theorem of hidden local symmetry for the vector mesons (KSRF (I) relation) in the $U(N)_{\mathrm{L}} \times U(N)_{\mathrm{R}} / U(N)_{\mathrm{V}}$ nonlinear chiral Lagrangian.
\end{abstract}

*Address after April 1, 1993 : Department of Physics, Kyoto University, Kyoto 606-01, Japan 
Hidden local symmetry (HLS) is a natural framework for describing the vector mesons in a manner consistent with the chiral symmetry of QCD [1]. The HLS Lagrangian yields at tree level a successful phenomenology for the pions and the $\rho$ mesons. By choosing a parameter $a=2$ in this HLS Lagrangian, we have[2]: 1) Universality of the $\rho$ meson coupling[3] $g_{\rho \pi \pi}=g$ ( $g$ : HLS gauge coupling); 2) KSRF relation [4] (version II) $\left.m_{\rho}^{2}=2 f_{\pi}^{2} g_{\rho \pi \pi}^{2} ; 3\right) \rho$ meson dominance of the electromagnetic form factor of the pion $g_{\gamma \pi \pi}=0[3]$.

Most remarkably, we further obtain an $a$-independent relation河 $g_{\rho} / g_{\rho \pi \pi}=2 f_{\pi}^{2}$, with $g_{\rho}$ being the strength of $\rho-\gamma$ mixing. This is actually another version of the celebrated KSRF relation (KSRF I) and is a decisive test of the HLS in the hadron physics. Since it follows from the symmetry structure alone, it was conjectured [5] to be a low energy theorem valid at zero momenta for any Lagrangian possessing the HLS and was further proved [6] at tree level. If it is indeed a genuine low energy theorem surviving the loop corrections, analogues of such a relation would also be useful for strongly coupled Higgs models or models of the dynamical electroweak symmetry breaking. Actually this HLS Lagrangian can be straightforwardly applied to such models[7].

Quite recently, it was shown in Landau gauge [8] that the above "low energy theorem" as well as the $a=2$ tree-level results at zero momenta is not altered by the one-loop corrections, thus strongly suggesting that it may indeed be a true low energy theorem[9]. These results are actually the relations coming from $O\left(p^{2}\right)$ operators or dimension-2 operators (counting only dimensions of the gauge fields and the derivatives).

In this paper we shall prove that the above "low energy theorem" of HLS actually holds at any loop order, based on the Ward-Takahashi (WT) identity for the BecchiRouet-Stora (BRS) symmetry. By restricting ourselves to the dimension-2 operators mentioned above, we can prove it by mathematical induction in quite the same way as the renormalizability proof for gauge theories [10] and two-dimensional nonlinear 
sigma models 11. In the following we assume that there exists a gauge invariant regularization (for example, the dimensional regularization).

Let us start with the $G_{\text {global }} \times H_{\text {local }}$ "linear model", with $G=U(N)_{\mathrm{L}} \times U(N)_{\mathrm{R}}$ and $H=U(N)_{\mathrm{V}}$. The Lagrangian is given by[2]

$$
\begin{aligned}
\mathcal{L}= & \mathcal{L}_{\mathrm{A}}+a \mathcal{L}_{\mathrm{V}}+\mathcal{L}_{\text {kin }}\left(V_{\mu}\right) \\
& \mathcal{L}_{\mathrm{V}}=-\frac{f_{\pi}^{2}}{4} \operatorname{tr}\left[\left(D_{\mu} \xi_{\mathrm{L}} \cdot \xi_{\mathrm{L}}^{\dagger} \mp D_{\mu} \xi_{\mathrm{R}} \cdot \xi_{\mathrm{R}}^{\dagger}\right)^{2}\right],
\end{aligned}
$$

where $a$ is a constant and $\mathcal{L}_{\text {kin }}\left(V_{\mu}\right)$ denotes the kinetic term of the hidden gauge boson (vector meson). Here $\xi_{\mathrm{L}}(x)$ and $\xi_{\mathrm{R}}(x)$ are two $U(N)$-matrix valued variables, which transform as $\xi_{\mathrm{L}, \mathrm{R}}(x) \rightarrow \xi_{\mathrm{L}, \mathrm{R}}^{\prime}(x)=h(x) \xi_{\mathrm{L}, \mathrm{R}}(x) g_{\mathrm{L}, \mathrm{R}}^{\dagger}$, where $h(x) \in H_{\text {local }}$ and $g_{\mathrm{L}, \mathrm{R}} \in G_{\text {global }}$. These variables are parameterized as $\xi_{\mathrm{L}, \mathrm{R}}(x) \equiv \exp \left[i \phi_{\mathrm{L}, \mathrm{R}}^{a}(x) T_{a}\right]$, where $T_{a}$ denotes the $U(N)$ generator. The covariant derivatives of $\xi_{\mathrm{L}, \mathrm{R}}(x)$ are defined by

$$
D_{\mu} \xi_{\mathrm{L}} \equiv \partial_{\mu} \xi_{\mathrm{L}}-i V_{\mu} \xi_{\mathrm{L}}+i \xi_{\mathrm{L}} \mathcal{V}_{\mathrm{L} \mu}
$$

and similar one with replacement $\mathrm{L} \leftrightarrow \mathrm{R}, \mathcal{V}_{\mathrm{L} \mu} \leftrightarrow \mathcal{V}_{\mathrm{R} \mu}$, where $V_{\mu}\left(\equiv V_{\mu}^{a} T_{a}\right)$ is the hidden gauge boson field and $\mathcal{V}_{\mathrm{L} \mu}$ and $\mathcal{V}_{\mathrm{R} \mu}$ denote the external gauge fields gauging the $G_{\text {global }}$ symmetry.

Now we consider the loop effects of the model. Let us take a covariant gauge condition for the HLS, and introduce the corresponding gauge-fixing and FaddeevPopov (FP) terms:

$$
\mathcal{L}_{G F}+\mathcal{L}_{F P}=B^{a} \partial^{\mu} V_{\mu}^{a}+\frac{1}{2} \alpha B^{a} B^{a}+i \bar{C}^{a} \partial^{\mu} D_{\mu} C^{a}
$$

where $B^{a}$ is the Nakanishi-Lautrap (NL) field and $C^{a}\left(\bar{C}^{a}\right)$ the FP ghost (anti-ghost) field. In this paper we do not consider the radiative corrections due to the external gauge fields $\mathcal{V}_{\mu}^{i} \equiv\left(\mathcal{V}_{\mathrm{L} \mu}^{a}, \mathcal{V}_{\mathrm{R} \mu}^{a}\right)$, so that we need not introduce the gauge-fixing terms for $\mathcal{V}_{\mu}$. Then, the corresponding ghost fields $\mathcal{C}^{i} \equiv\left(\mathcal{C}_{\mathrm{L}}^{a}, \mathcal{C}_{\mathrm{R}}^{a}\right)$ are non-propagating.

The infinitesimal form of the $G_{\text {global }} \times H_{\text {local }}$ transformation of the Nambu-Goldstone $(\mathrm{NG})$ field $\phi^{i} \equiv\left(\phi_{L}^{a}, \phi_{R}^{a}\right)$ is given by $\delta \phi^{i}=\theta^{a} W_{a}^{i}(\phi)+\vartheta^{j} \mathcal{W}_{j}^{i}(\phi)\left(\equiv \theta^{A} \boldsymbol{W}_{A}^{i}(\phi)\right)$, where 
$A$ denotes a set $(a, i)$ of labels of $H_{\text {local }}$ and $G_{\text {global }}$. Accordingly, the BRS transformation of the NG fields $\phi^{i}$, the gauge fields $\boldsymbol{V}_{\mu}^{A} \equiv\left(V_{\mu}^{a}, \mathcal{V}_{\mu}^{i}\right)$ and the FP ghost fields $C^{A} \equiv\left(C^{a}, \mathcal{C}^{i}\right)$ are respectively given by

$$
\begin{aligned}
\delta_{\mathrm{B}} \phi^{i} & =\boldsymbol{C}^{A} \hat{\boldsymbol{W}}_{A} \phi^{i} \quad\left(\hat{\boldsymbol{W}}_{A} \equiv \boldsymbol{W}_{A}^{i}(\phi) \frac{\partial}{\partial \phi^{i}}\right), \\
\delta_{\mathrm{B}} \boldsymbol{V}_{\mu}^{A} & =\partial_{\mu} \boldsymbol{C}^{A}+\boldsymbol{V}_{\mu}^{B} \boldsymbol{C}^{C} f_{B C}{ }^{A} \\
\delta_{\mathrm{B}} \boldsymbol{C}^{A} & =-\frac{1}{2} \boldsymbol{C}^{B} \boldsymbol{C}^{C} f_{B C}{ }^{A} .
\end{aligned}
$$

We define the dimension of the fields as $\operatorname{dim}\left[\phi_{L, R}^{a}\right]=0, \operatorname{dim}\left[\boldsymbol{V}_{\mu}^{A}\right]=1$. It is also convenient to assign the following dimensions to the FP-ghosts: $\operatorname{dim}\left[\boldsymbol{C}^{A}\right]=$ $0, \operatorname{dim}\left[\bar{C}^{a}\right]=2$. Then the BRS transformation does not change the dimension. According to the above dimension counting, we may divide the Lagrangian Eq.(1) plus Eq.(3) into two parts: 1) dimension-2 part $\mathcal{L}_{\mathrm{A}}+a \mathcal{L}_{\mathrm{V}}$ and 2) dimension-4 part $\mathcal{L}_{\text {kin }}\left(V_{\mu}\right)+\mathcal{L}_{\mathrm{GF}}+\mathcal{L}_{\mathrm{FP}}$. (We count the dimension of the fields and derivatives only, hereafter.)

Now, we consider the quantum correction to this system at any loop order, and prove the following proposition.

Proposition : As far as the dimension-2 operators are concerned, all the quantum corrections, including the finite parts as well as the divergent parts, can be absorbed into the original dimension-2 Lagrangian $\mathcal{L}_{\mathrm{A}}+a \mathcal{L}_{\mathrm{V}}$ by a suitable redefinition (renormalization) of the parameters $a, f_{\pi}^{2}$, and the fields $\phi^{i}, V_{\mu}^{a}$.

This implies that the tree-level dimension-2-Lagrangian, with the parameters and fields substituted by the "renormalized" ones, already describes the exact action at any loop order, and therefore that all the "low energy theorems" derived from it receive no quantum corrections at all.

We prove the above proposition in the same way as the renormalizability proof for gauge theories [10] and two dimensional nonlinear sigma models [1]. We can write down the WT identity for the effective action $\Gamma$. The NL fields $B^{a}$ and the FP antighost fields $\bar{C}^{a}$ can be eliminated from $\Gamma$ by using their equations of motion as usual. 
Then the tree level action $S=\Gamma_{\text {tree }}$ reads

$$
\begin{gathered}
S[\Phi, \mathbf{K} ; \boldsymbol{a}]=S_{2}[\phi, \boldsymbol{V}]+S_{4}[\Phi, \mathbf{K}], \\
S_{2}[\phi, \boldsymbol{V}]=\int d^{4} x\left(a_{\perp} \mathcal{L}_{\mathrm{A}}(\phi, \boldsymbol{V})+a_{\|} \mathcal{L}_{\mathrm{V}}(\phi, \boldsymbol{V})\right), \\
S_{4}[\Phi, \mathbf{K}]=\int d^{4} x\left(\mathcal{L}_{\mathrm{kin}}\left(V_{\mu}\right)+\mathbf{K} \cdot \delta_{\mathrm{B}} \Phi\right),
\end{gathered}
$$

where $\Phi \equiv\left(\phi^{i}, \boldsymbol{V}_{\mu}^{A}, \boldsymbol{C}^{A}\right)$ are the field variables and $\mathbf{K} \equiv\left(K_{i}, \boldsymbol{K}_{A}^{\mu}, \boldsymbol{L}_{A}\right)\left(\boldsymbol{K}_{A}^{\mu} \equiv\right.$ $\left.\left(K_{a}^{\mu}, \mathcal{K}_{i}^{\mu}\right), \boldsymbol{L}_{A} \equiv\left(L_{a}, \mathcal{L}_{i}\right)\right)$ denote the BRS source fields. We have rewritten $a$ and $f_{\pi}^{2}$ as $a f_{\pi}^{2} \rightarrow a_{\|} f_{\pi}^{2}$ and $f_{\pi}^{2} \rightarrow a_{\perp} f_{\pi}^{2}$, so that the renormalization of $a$ and $f_{\pi}^{2}$ corresponds to that of $\boldsymbol{a} \equiv\left(a_{\|}, a_{\perp}\right)$. According to the dimension assignment of the fields, the dimension of the above BRS source fields $\mathbf{K}$ is given by $\operatorname{dim}\left[K_{i}\right]=\operatorname{dim}\left[\boldsymbol{L}_{A}\right]=4$ and $\operatorname{dim}\left[\boldsymbol{K}_{A}^{\mu}\right]=3$.

The WT identity for the effective action $\Gamma$ is given by

$$
\Gamma * \Gamma=0
$$

where the $*$ operation is defined by

$$
F * G=(-)^{\Phi} \frac{\overleftarrow{\delta} F}{\delta \Phi} \frac{\delta G}{\delta \mathbf{K}}-(-)^{\Phi} \frac{\overleftarrow{\delta} F}{\delta \mathbf{K}} \frac{\delta G}{\delta \Phi}
$$

for arbitrary functionals $F[\Phi, \mathbf{K}]$ and $G[\Phi, \mathbf{K}]$. (Here the symbols $\delta$ and $\overleftarrow{\delta}$ denote the derivatives from the left and right, respectively, and $(-)^{\Phi}$ denotes +1 or -1 when $\Phi$ is bosonic or fermionic, respectively.)

The effective action is calculated in the loop expansion: $\Gamma=S+\hbar \Gamma^{(1)}+\hbar^{2} \Gamma^{(2)}+\cdots$. The $\hbar^{n}$ term $\Gamma^{(n)}$ contains contributions not only from the genuine $n$-loop diagrams but also from the lower loop diagrams including the counter terms. We can expand the $n$-th term $\Gamma^{(n)}$ according to the dimension:

$$
\Gamma^{(n)}=\Gamma_{0}^{(n)}[\phi]+\Gamma_{2}^{(n)}[\phi, \boldsymbol{V}]+\Gamma_{4}^{(n)}[\Phi, \mathbf{K}]+\cdots
$$

Here again we are counting the dimension only of the fields and derivatives. The first dimension-0 term $\Gamma_{0}^{(n)}$ can contain only the dimensionless field $\phi^{i}$ without derivatives. 
The two dimensions of the second term $\Gamma_{2}^{(n)}$ is supplied by derivative and/or the gauge field $\boldsymbol{V}_{\mu}^{A}$. The BRS source field $\mathbf{K}$ carries dimension 4 or 3 , and hence it can appear only in $\Gamma_{4}^{(n)}$ and beyond: the dimension- 4 term $\Gamma_{4}^{(n)}$ is at most linear in $\mathbf{K}$, while the dimension- 6 term $\Gamma_{6}^{(n)}$ can contain a quadratic term in $K_{a}^{\mu}$, the BRS source of the hidden gauge boson $V_{\mu}^{a}$. To calculate $\Gamma^{(n)}$, we need to use the "bare" action, $\left(S_{0}\right)_{n}=S\left[\left(\Phi_{0}\right)_{n},\left(\mathbf{K}_{0}\right)_{n} ;\left(\boldsymbol{a}_{0}\right)_{n}\right]$, where the $n$-th loop order "bare" fields $\left(\Phi_{0}\right)_{n},\left(\mathbf{K}_{0}\right)_{n}$ and parameters $\left(\boldsymbol{a}_{0}\right)_{n}$ are given by

$$
\begin{aligned}
\left(\Phi_{0}\right)_{n} & =\Phi+\hbar \delta \Phi^{(1)}+\cdots+\hbar^{n} \delta \Phi^{(n)}, \\
\left(\mathbf{K}_{0}\right)_{n} & =\mathbf{K}+\hbar \delta \mathbf{K}^{(1)}+\cdots+\hbar^{n} \delta \mathbf{K}^{(n)}, \\
\left(\boldsymbol{a}_{0}\right)_{n} & =\boldsymbol{a}+\hbar \delta \boldsymbol{a}^{(1)}+\cdots+\hbar^{n} \delta \boldsymbol{a}^{(n)} .
\end{aligned}
$$

Let us now prove the following by mathematical induction with respect to the loop expansion parameter $n$ :

1) $\Gamma_{0}^{(n)}(\phi)=0$.

2) By choosing suitably the $n$-th order counter terms $\delta \Phi^{(n)}, \delta \mathbf{K}^{(n)}$ and $\delta \boldsymbol{a}^{(n)}$, $\Gamma_{2}^{(n)}[\phi, A]$ and the $\mathbf{K}$-linear terms in $\Gamma_{4}^{(n)}[\Phi, \mathbf{K}]$ can be made vanish; $\Gamma_{2}^{(n)}[\phi, \boldsymbol{V}]=$ $\left.\Gamma_{4}^{(n)}[\Phi, \mathbf{K}]\right|_{\mathbf{K} \text {-linear }}=0$.

3) The field reparameterization (renormalization) $(\Phi, \mathbf{K}) \rightarrow\left(\left(\Phi_{0}\right)_{n},\left(\mathbf{K}_{0}\right)_{n}\right)$ is a "canonical" transformation which leaves the $*$ operation invariant.

Suppose that the above statements are satisfied for the $(n-1)$-th loop order effective action $\Gamma^{(n-1)}$. We calculate, for the moment, the $n$-th loop effective action $\Gamma^{(n)}$ using the $(n-1)$-th loop level "bare" action $\left(S_{0}\right)_{n-1}$, i.e., without $n$-th loop counter terms. We expand the $\hbar^{n}$ terms in the WT identity $S * \Gamma^{(n)}=-\frac{1}{2} \sum_{l=1}^{n-1} \Gamma^{(l)} *$ $\Gamma^{(n-l)}$ according to the dimensions like in Eq.(8). Then using the above induction assumption, we find: $S_{4} * \Gamma_{0}^{(n)}+S_{2} * \Gamma_{2}^{(n)}=0(\operatorname{dim} 0), S_{4} * \Gamma_{2}^{(n)}+S_{2} * \Gamma_{4}^{(n)}=0(\operatorname{dim}$ 2) and $S_{4} * \Gamma_{4}^{(n)}+S_{2} * \Gamma_{6}^{(n)}=0(\operatorname{dim} 4)$. These three renormalization equations give 
enough information for determining possible forms of $\Gamma_{0}^{(n)}, \Gamma_{2}^{(n)}$ and $\left.\Gamma_{4}^{(n)}\right|_{\mathbf{K} \text {-linear }}$ (the $\mathbf{K}$-linear term in $\Gamma_{4}^{(n)}$ ) which we are interested in.

First, the dimension-0 part of the renormalization equation reads $\delta_{\mathrm{B}} \Gamma_{0}^{(n)}=0$. Since there are no invariants containing no derivatives, we can immediately conclude $\Gamma_{0}^{(n)}=0$, and hence our statement 1) follows.

Next, we solve the dimension- 2 and the dimension-4 parts of the above renormalization equations. It is convenient to define the BRS-like transformation $\delta_{\Gamma}^{\prime}$ on the fields $\Phi$ by $\delta_{\Gamma}^{\prime} \equiv\left(\delta \Gamma_{4}^{(n)} / \delta \mathbf{K}\right) \frac{\delta}{\delta \Phi}$. Then these equations read

$$
\begin{aligned}
& \delta_{\mathrm{B}} \Gamma_{2}^{(n)}+\delta_{\Gamma}^{\prime} S_{2}=0, \\
& \delta_{\mathrm{B}} \Gamma_{4}^{(n)}+\delta_{\Gamma}^{\prime} S_{4}+\frac{\delta \Gamma_{6}^{(n)}}{\delta \mathbf{K}} \frac{\delta S_{2}}{\delta \Phi}=0 .
\end{aligned}
$$

A tedious but straightforward analysis [12 of the K-linear term in Eq.(11) determines the general form of the $\left.\Gamma_{4}^{(n)}\right|_{\mathbf{K} \text {-linear }}$ and $\left.\Gamma_{6}^{(n)}\right|_{\mathbf{K} \text {-quadratic }}$ terms: the solution for $\left.\Gamma_{4}^{(n)}\right|_{\mathbf{K} \text {-linear }}$ or equivalently $\delta_{\Gamma}^{\prime}$ is given by

$$
\begin{aligned}
& \delta_{\Gamma}^{\prime} C^{a}=\beta \delta_{\mathrm{B}} C^{a}, \\
& \delta_{\Gamma}^{\prime} \phi^{i}=\left\{C^{a}\left(\left[\hat{W}_{a}, \hat{F}\right]+\beta \hat{W}_{a}\right)+\mathcal{C}^{j}\left[\hat{\mathcal{W}}_{j}, \hat{F}\right]\right\} \phi^{i}, \\
& \delta_{\Gamma}^{\prime} V_{\mu}^{a}=\alpha \partial_{\mu} C^{a}+\beta \delta_{\mathrm{B}} V_{\mu}^{a}+\gamma \delta_{\mathrm{B}}\left(V_{\mu}^{a}-\tilde{\mathcal{V}}_{\mu}^{a}\right),
\end{aligned}
$$

where $\alpha, \beta$ and $\gamma$ are constants, $\tilde{\mathcal{V}}_{\mu} \equiv \xi_{\mathrm{L}} \mathcal{V}_{\mathrm{L} \mu} \xi_{\mathrm{L}}^{\dagger}-i \partial_{\mu} \xi_{\mathrm{L}} \cdot \xi_{\mathrm{L}}^{\dagger}+(\mathrm{L} \leftrightarrow \mathrm{R})$ and $\hat{F} \equiv$ $F^{i}(\phi) \partial / \partial \phi^{i}$, with $F^{i}(\phi)$ being a certain dimension-0 function. Note that $\delta_{\Gamma}^{\prime} \mathcal{V}_{\mu}^{i}=$

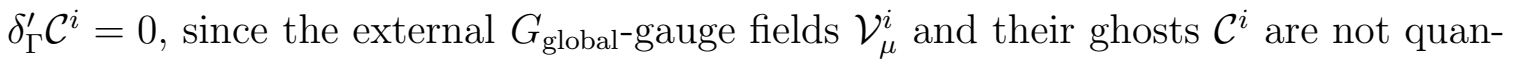
tized and hence their BRS source fields $\mathcal{K}_{i}^{\mu}$ and $\mathcal{L}_{i}$ appear only in the tree action.

Using $\delta_{\Gamma}^{\prime}$ thus obtained, we next solve the above WT identity Eq.(10) and easily find

$$
\Gamma_{2}^{(n)}=A_{2 \mathrm{GI}}[\phi, \boldsymbol{V}]-\left(\hat{F} S_{2}+\alpha V_{\mu}^{a} \frac{\delta}{\delta V_{\mu}^{a}} S_{2}\right),
$$

where $A_{2 \mathrm{GI}}$ is a dimension-2 gauge-invariant function of $\phi^{i}$ and $\boldsymbol{V}_{\mu}^{A}$. 
The solutions are combined into a simple form

$$
\Gamma_{2}^{(n)}+\left.\Gamma_{4}^{(n)}\right|_{\mathbf{K} \text {-linear }}=A_{2 \mathrm{GI}}[\phi, \boldsymbol{V}]-S * Y
$$

up to irrelevant terms (dimension-6 or $\mathbf{K}$-independent dimension-4 terms), where the functional $Y$ is given by

$$
\begin{aligned}
Y=\int d^{4} x[ & K_{i} F^{i}(\phi)+\alpha K_{a}^{\mu} V_{\mu}^{a} \\
& \left.+\beta L_{a} C^{a}+\gamma f_{a b c} K_{a}^{\mu} K_{b \mu} C^{c}\right] .
\end{aligned}
$$

Now, we prove our statements 2) and 3) in the above. We have calculated the above effective action $\Gamma^{(n)}$ without using $n$-th loop level counter terms $\delta \Phi^{(n)}, \delta \mathbf{K}^{(n)}$ and $\delta \boldsymbol{a}^{(n)}$. If we include those, we have the additional contribution given by

$$
\Delta \Gamma^{(n)}=\delta \Phi^{(n)} \frac{\delta S}{\delta \Phi}+\delta \mathbf{K}^{(n)} \frac{\delta S}{\delta \mathbf{K}}+\delta \boldsymbol{a}^{(n)} \frac{\partial S}{\partial \boldsymbol{a}},
$$

where $S[\Phi, \mathbf{K} ; \boldsymbol{a}]$ is the tree-level action. So the true $n$-th loop level effective action is given by $\Gamma^{(n)}+\Delta \Gamma^{(n)} \equiv \Gamma_{\text {total }}^{(n)}$. The tree-level action $S_{2}$ is the most general gauge-

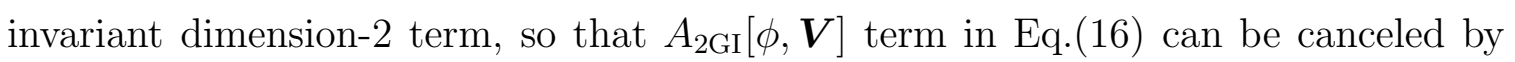
suitably chosen counter terms, $\delta \boldsymbol{a}^{(n)} \frac{\partial S}{\partial \boldsymbol{a}}$. The second term $-S * Y$ term in Eq.(16) just represents a "canonical transformation" of $S$ generated by $-Y$. Therefore we choose the $n$-th order field counter terms $\delta \Phi^{(n)}$ and $\delta \mathbf{K}^{(n)}$ to be equal to the canonical transformations of $\Phi$ and $\mathbf{K}$ generated by $+Y ; \delta \Phi^{(n)}=\Phi * Y, \delta \mathbf{K}^{(n)}=\mathbf{K} * Y$. Then the first and the second terms in Eq. (18) just give $S * Y$ and precisely cancel the second term in Eq.(16). Thus we have completed the proof of our statements 2) and $3)$.

Some comments are in order:

1. Our conclusion in this paper remains unaltered even if the action $S$ contains other dimension-4 or higher terms, as far as they respect the symmetry. This is because we needed just $(S * \Gamma)_{2}$ and $\left.(S * \Gamma)_{4}\right|_{\mathbf{K} \text {-linear }}$ parts in the WT identity to which only $S_{2}$ and $\mathbf{K}$-linear part of $S_{4}$ can contribute. 
2. When we regard this HLS model as a low energy effective theory of QCD, we must take account of the anomaly and the corresponding Wess-Zumino-Witten term $\Gamma_{\text {WZW }}$. The WT identity now reads $\Gamma * \Gamma=$ (anomaly). However, the RHS is saturated already at the tree level in this effective Lagrangian and so the WT identity at loop levels, which we need, remains the same as before. The WZW term $\Gamma_{\text {WZW }}$ or any other intrinsic-parity-odd terms 13 in $S$ are of dimension-4 or higher and hence do not change our conclusion as explained above.

3. We have shown in the covariant gauges that our tree-level dimension-2 action $\int d^{4} x\left(\mathcal{L}_{\mathrm{A}}+a \mathcal{L}_{\mathrm{V}}\right)$, if written in terms of renormalized parameters and fields, already gives the exact action $\Gamma_{2}$ including all the loop effects. This form of the effective action (in particular the $\mathcal{L}_{\mathrm{V}}$ part) implies that the previously derived relation[5, 6]

$$
\left.\frac{g_{V}\left(p^{2}\right)}{g_{V \pi \pi}\left(p^{2}, p_{\pi_{1}}^{2}=p_{\pi_{2}}^{2}=0\right)}\right|_{p^{2}=0}=2 f_{\pi}^{2}
$$

is actually an exact low energy theorem valid at any loop order. Of course, this theorem concerns off-shell quantities at $p^{2}=0$ of the vector field momentum $p$, and hence is not physical as it stands. However, suppose that the vector mass $m_{V}^{2}=a g^{2} f_{\pi}^{2}$ is sufficiently small compared with the characteristic energy scale $\Lambda^{2}$ of the system, which is customarily taken as $\Lambda^{2} \sim 16 \pi^{2} f_{\pi}^{2}$. Then we expect that the on-shell value of $g_{V} / g_{V \pi \pi}$ at $p^{2}=m_{V}^{2}$ can deviate from the LHS of Eq. (19) only by a quantity of order $m_{V}^{2} / \Lambda^{2} \sim a g^{2} / 16 \pi^{2}$, since the contributions of the dimension- 4 or higher terms in the effective action $\Gamma$ (again representing all the loop effects) are suppressed by a factor of $p^{2} / \Lambda^{2}$ at least. Therefore as far as the vector mass is light, namely, when either $a$ or $g^{2} / 16 \pi^{2}$ is small, our theorem is truly a physical one 14]. In the actual world of QCD, the $\rho$ meson mass is not so light $\left(a g^{2} / 16 \pi^{2} \sim 1 / 2\right)$ so that the situation becomes a bit obscure. Nevertheless, the fact that the KSRF (I) relation $g_{\rho} / g_{\rho \pi \pi}=2 f_{\pi}^{2}$ holds on the $\rho$ mass shell with good accuracy strongly suggests that the $\rho$ meson is the hidden gauge field and the KSRF (I) relation is a physical manifestation of our low energy theorem. 
4. In this connection we should comment on the gauge choice. In the covariant gauges which we adopted here, the $G_{\text {global }}$ and $H_{\text {local }}$ BRS symmetries are separately preserved. Accordingly, the $V_{\mu}$ field is multiplicatively renormalized (recall that $\delta V_{\mu}^{(n)}=V_{\mu} * Y=\alpha V_{\mu}$ ), and the above (off-shell) low energy theorem Eq.(19) holds. However, if we adopt $R_{\xi}$-gauges (other than Landau gauge), these properties are violated; for instance, $\phi \partial_{\mu} \phi$ or the external gauge field $\mathcal{V}_{\mu}$ gets mixed with our $V_{\mu}$ through the renormalization, and our off-shell low energy theorem Eq.(19) is violated. This implies that the $V_{\mu}$ field in the $R_{\xi}$ gauge generally does not give a smooth off-shell extrapolation; indeed, in $R_{\xi}$ gauge with gauge parameter $\alpha \equiv 1 / \xi$, the correction to $g_{\rho} / g_{\rho \pi \pi}$ by the extrapolation from $p^{2}=m_{\rho}^{2}$ to $p^{2}=0$ is seen to have a part proportional to $\alpha g^{2} / 16 \pi^{2}$, which diverges when $\alpha$ becomes very large. Thus, in particular, the unitary gauge[15], which corresponds to $\alpha \rightarrow \infty$, gives an ill-defined off-shell field.

5. Our argument is free from infrared divergences at least in Landau gauge. This can be seen as follows. In this gauge the propagators of the NG bosons, the hidden gauge bosons and the FP ghosts (after rescaling the FP anti-ghost $\bar{C}$ into $f_{\pi}^{2} \bar{C}$ ) are all proportional to $1 / f_{\pi}^{2}$ in the infrared region. Therefore, a general $L$-loop diagram, which includes $V_{4}$ dimension- 4 vertices and $K$ BRS source vertices, yields an amplitude proportional to $\left(1 / f_{\pi}^{2}\right)^{\left(L-1+V_{4}+K\right)}$ 16. Thus, from dimensional consideration we see that there is no infrared contribution to $\Gamma_{0}^{(n)}[\phi], \Gamma_{2}^{(n)}[\phi, \boldsymbol{V}]$ and $\left.\Gamma_{4}^{(n)}[\Phi, \mathbf{K}]\right|_{\mathbf{K} \text {-linear }}$. In other covariant gauges, there appears a dipole ghost in the vector propagator, which is to be defined by a suitable regularization.

We would like to thank Volodya Miransky and Masaharu Tanabashi for stimulating discussions. T. K. is supported in part by the Grant-in-Aid for Scientific Research (\#04640292) from the Ministry of Education, Science and Culture. K. Y. is supported in part by the Takeda Science Foundation and the Ishida Foundation, and also by the International Collaboration Program of the Japan Society for Promotion of Science. 


\section{References}

[1] For a review, M. Bando, T. Kugo and K. Yamawaki, Phys. Rep. 164, 217 (1988).

[2] M. Bando, T. Kugo, S. Uehara, K. Yamawaki and T. Yanagida, Phys. Rev. Lett. 54, 1215 (1985).

[3] J.J. Sakurai, Currents and mesons (Chicago Univ. Press, Chicago, 1969).

[4] K. Kawarabayashi and M. Suzuki, Phys. Rev. Lett. 16, 255 (1966); Riazuddin and Fayyazuddin, Phys. Rev. 147, 1071 (1966).

[5] M. Bando, T. Kugo and K. Yamawaki, Nucl. Phys. B259, 493 (1985).

[6] M. Bando, T. Kugo and K. Yamawaki, Prog. Theor. Phys. 73, 1541 (1985).

[7] R. Casalbuoni, S. de Curtis, D. Dominici and R. Gatto, Phys. Lett. B155, 95 (1985); Nucl. Phys. B282, 235 (1987).

[8] M. Harada and K. Yamawaki, Phys. Lett. B297, 151 (1992).

[9] We are actually discussing the "low energy theorem" relating the off-shell quantities. Thus they depend on the definition of the $\rho$ field and hence on the gauge choice. We shall return to this problem later.

[10] C. Becchi, A. Rouet and R. Stora, Ann. of Phys. (N.Y.) 98, 287 (1976).

[11] A. Blasi and R. Collina, Nucl. Phys. B258, 204 (1987); Phys. Lett. B200, 98 (1988).

[12] The detailed analysis is given in the paper ("Low Energy Theorems of Hidden Local Symmetries", M. Harada, T. Kugo and K. Yamawaki, KUNS-1179/DPNU93-2) for the models with arbitrary compact groups $G$ and $H(\subseteq G)$. 
[13] T. Fujiwara, T. Kugo, H. Terao, S. Uehara and K. Yamawaki, Prog. Theor. Phys. 73,926 (1985).

[14] It is interesting to note that the "vector limit" (H. Georgi, Phys. Rev. Lett. 63, 1917 (1989); Nucl. Phys. B331, 311 (1990).) realizes this light vector meson limit.

[15] In the unitary gauge our HLS Lagrangian coincides with the Weinberg's old Lagrangian for the $\rho$ meson (S. Weinberg, Phys. Rev. 166, 1568 (1968).).

[16] This counting is similar to that in the nonlinear chiral Lagrangian (S. Weinberg, Physica 96A, 327 (1979)). 